\title{
Messenger RNA sequencing reveals similar mechanisms between neonatal and acute respiratory distress syndrome
}

\author{
HUA MEI $^{1}$, YUHENG ZHANG ${ }^{1}$, CHUNZHI LIU $^{1}$, YAYU ZHANG ${ }^{1}$, CHUNLI LIU $^{1}$, DAN SONG ${ }^{1}$, \\ CHUN XIN $^{1}$, JING WANG ${ }^{1}$, JONATHAN JOSEPHS-SPAULDING ${ }^{2}$, YAN ZHU $^{2}$ and FENG TANG ${ }^{2}$ \\ ${ }^{1}$ Department of Pediatrics, The Affiliated Hospital of Inner Mongolia Medical University, Huhehaote, \\ Inner Mongolia 010050; ${ }^{2}$ Beijing Amorlife Pharma Services Co., Ltd., Beijing 100176, P.R. China
}

Received May 15, 2017; Accepted September 22, 2017

DOI: $10.3892 / \mathrm{mmr} .2017 .7891$

\begin{abstract}
Hypoxemia and hypercarbia resulting from a lack of surfactant is considered to be the primary mechanism underlying neonatal respiratory distress syndrome (NRDS). Surfactant replacement therapy may mitigate the symptoms of the disease by decreasing the surface tension of alveoli and facilitating inflation. However, surfactant serves an additional role in immunological processes. Therefore, it may be hypothesized that mechanisms of NRDS involving surfactant exert additional functions to promoting alveolar inflation. Using peripheral blood obtained from mature infants with and without NRDS, in tandem with mRNA sequencing (mRNA-seq) analysis, the present study identified that, while cell cycle regulation and alveolar surfactants serve a role in deterring the further onset of NRDS, innate and pathogen-induced responses of the immune system are among the most important factors in the pathology. The present study illustrated the regulatory importance of these immune pathways in response to alterations in the expression of gene families, particularly in perpetual lung injury leading to NRDS. Notably, data collected from the mRNA-seq analysis revealed similar mechanisms between NRDS and acute respiratory distress syndrome, a clinical phenotype precipitated by the manifestation of a severe form of lung injury due to numerous lung insults, implying that similar therapies may be applied to treat these two diseases.
\end{abstract}

\section{Introduction}

Neonatal respiratory distress syndrome (NRDS), also termed hyaline membrane disease, is one of the most prevalent causes of morbidity and mortality in newborns (1). The oxygen

Correspondence to: Dr Hua Mei, Department of Pediatrics, The Affiliated Hospital of Inner Mongolia Medical University, 1 North Tongdao Road, Huhehaote, Inner Mongolia 010050, P.R. China E-mail:meihuayani@sina.com

Key words: mRNA sequencing, neonatal respiratory distress syndrome, acute respiratory distress syndrome, mechanism diffusion efficiency through the alveoli-capillary exchange barrier is impacted by the dysregulation of numerous factors, leading to the pathophysiology of NRDS (2). Cellular stress at the gas-blood level is associated with alterations in alveolar surfactant proteins (3) and the upregulation of numerous innate immune responses of pro-inflammatory cytokines to foreign challenges (4). A lack of surfactant may drive the pathogenesis towards NRDS, while surfactant replacement therapy may mitigate symptoms of the disease by decreasing the surface tension of alveoli and facilitating their inflation $(5,6)$. However, surfactant serves an additional role in immunological processes. A recent study demonstrated that applying clinical surfactant for patients led to a stronger response to challenges from foreign microbiota (7). Additionally, animal-derived surfactant applied for clinical use in humans has been demonstrated to be of use against lung pathogens and to mediate excess host damage from neutrophils (8). Therefore, it may be hypothesized that mechanisms that mechanisms of NRDS involving surfactant exert additional functions to promoting alveolar inflation.

Acute respiratory distress syndrome (ARDS) is a clinical phenotype precipitated by the manifestation of a severe form of lung injury due to numerous lung insults (9). The pathophysiological manifestation of ARDS is derived from a cascade that is triggered by the complex combination of risk factors, including asthma, sepsis, pneumonia, increased neutrophil presence in the lungs and other variables; accumulation of these numerous systemic factors forms the pathological endpoint of ARDS (10).

The present study used peripheral blood obtained from infants with $(n=4)$ or without $(n=2)$ NRDS in tandem with mRNA-sequencing (mRNA-seq) analysis to reveal various factors involved in the mechanisms of NRDS, and to compare onset mechanisms between NRDS and ARDS.

\section{Materials and methods}

Samples. The Ethics Committee of the Affiliated Hospital of Inner Mongolia Medical University (Huhehaote, China) approved the present study. All study participants provided written informed consent and were recruited at the Affiliated Hospital of Inner Mongolia Medical University between January 1 and June 30, 2016. 
A total of four infants with severe NRDS and two healthy infants were recruited for the present study. Subject characteristics are presented in Table I. The subjects were not related, and all of their relatives in the three most recent generations were residents in Inner Mongolia province and self-identified as Chinese Inner Mongolia race. NRDS was diagnosed according to the European Consensus Guidelines on the Diagnosis of NRDS in Preterm Infants 2010 (11). The criteria were as follows: Progressive deterioration of shortness of breath commencing at $12 \mathrm{~h}$ post-birth, with arterial partial pressure of oxygen $\left(\mathrm{PaO}_{2}\right)<50 \mathrm{mmHg}$ during inspiration accompanied by central cyanosis. Oxygen therapy was required to maintain $\mathrm{PaO}_{2}>50 \mathrm{mmHg}$. Chest X-ray (CXR) indicated NRDS specific signs, including ground glass opacity and air-filled bronchi. The control groups were defined by CXR demonstrating clear lung field without pulmonary infection signs. White blood cell counts and C-reactive protein values were normal, to exclude the possibility of any infection. Subjects with any of the following were excluded from the present study: i) Any serious congenital diseases, including complex congenital cardiac anomaly or diaphragm hernia; ii) inherited metabolic disorders, including phenylketonuria, congenital hypothyroidism or diabetes mellitus; iii) confirmed maternal infection history during the third trimester of pregnancy; iv) multigestation; v) maternal diabetes mellitus; or vi) neonatal asphyxia.

$m R N A$ extraction and sequencing. Total RNAs from 4 NRDS and 2 control subjects were isolated and quality controlled using protocols from Illumina, Inc. (San Diego, CA, USA). A total amount of $3 \mu \mathrm{g} \mathrm{RNA} /$ sample was used as the input material for library construction. Ribosomal RNA (rRNA) was removed using the Epicentre Ribo-zero rRNA Removal kit (Illumina, Inc.), according to the manufacturer's protocol. Strand-specific sequencing libraries were generated via the dUTP method using the resulting RNA with the NEB Next Ultra Directional RNA Library Prep kit for Illumina (New England BioLabs, Inc., Ipswich, MA, USA), according to the manufacturer's protocol. RNA-seq was performed on an Illumina Hiseq 2000 platform (Illumina, Inc.) and 100-bp paired-end reads were generated, according to the manufacturer's protocol.

RNA-seq data analysis. The adapter sequences were removed from the raw sequencing data and the individual libraries were converted to the FASTQ format. Sequence reads were aligned to the human genome (hg19) using TopHat2 (v2.0.9; ccb.jhu.edu/software/tophat/index.shtml) and the resulting alignment files were reconstructed using Cufflinks (v2.1.1; cole-trapnell-lab.github.io/cufflinks) (12) and Scripture (beta2; software.broadinstitute.org/software/scripture). The transcripts assembled by Cufflinks and Scripture were used for the identification of differentially expressed genes (DEGs). For mRNA analyses, the RefSeq database (build 37.3; www.ncbi.nlm.nih.gov/refseq) was selected for the annotation references. The read counts of each transcript were normalized to the length of the individual transcript and to the total mapped fragment counts in each sample, and expressed as fragments per kilobase of exon per million fragments mapped of mRNAs in each sample. mRNA differential expression analyses were applied to the NRDS and control groups. The P-value was calculated using R3.2.5 software (www.r-project.org).
Table I. Subject information.

\begin{tabular}{llcc}
\hline Sample ID & Sex & Mother & Gestational age, weeks \\
\hline Control 1 & Male & G2P2 & $38+3$ \\
Control 2 & Female & G3P3 & $38+3$ \\
NRDS1 & Male & G2P1 & $37+6$ \\
NRDS2 & Male & G3P1 & $37+4$ \\
NRDS3 & Female & G3P2 & 37 \\
NRDS4 & Female & G2P1 & $37+3$ \\
\hline
\end{tabular}

$\mathrm{G}$, gravida (the total number of pregnancies); $\mathrm{P}$, para (the total number of deliveries).

Table II. Primers used for RT-qPCR.

\begin{tabular}{|c|c|}
\hline Gene & Primer \\
\hline \multirow[t]{2}{*}{ PIP4K2B } & Forward: ATTGCACTGGAGACCAGCAA \\
\hline & Reverse: GTACGCACAAAAGACTGGCG \\
\hline \multirow[t]{2}{*}{ FCAR } & Forward: GACCACCCTCCTGTGTCTTG \\
\hline & Reverse: GGATGGAGTCGTAGCACCTG \\
\hline \multirow[t]{2}{*}{ MLLT6 } & Forward: GTGGGCCATGGCAGAAGTAG \\
\hline & Reverse: CCCTGATTCAAAGCCCCGAA \\
\hline \multirow{2}{*}{ DDX52 } & Forward: TGCGATCAACTACTTCGGCA \\
\hline & Reverse: ATCGCAGCCATGGACGTTTA \\
\hline \multirow[t]{2}{*}{ AC010970 } & Forward: TGTCAGCACTCCCAACAGAC \\
\hline & Reverse: GTCCCTCATGGCCACAAGTT \\
\hline \multirow[t]{2}{*}{ APBA2 } & Forward: ACTGGGACCGCTACAGTACA \\
\hline & Reverse: ATCCAGACTGTCAGCATCGC \\
\hline \multirow{2}{*}{ BRD2 } & Forward: GTCTACCGATTCCCACCTCG \\
\hline & Reverse: GCCAAGATGGCTGTAGGTGT \\
\hline \multirow[t]{2}{*}{ HLA-DQA1 } & Forward: CTGACAAACATCGCTGTGCT \\
\hline & Reverse: GAAGCACCAACTGAACGCAG \\
\hline \multirow[t]{2}{*}{ MDGA1 } & Forward: CTCCGAGTACCCCACAGCTA \\
\hline & Reverse: CATGGATCCCCAAAGTTGCAG \\
\hline \multirow[t]{2}{*}{ WASF3 } & Forward: CTTTTAGGGAACCCGCTGGA \\
\hline & Reverse:TAGAGCGAACATGGACGACAG \\
\hline \multirow[t]{2}{*}{ GAPDH } & Forward: TGCACCACCAACTGCTTAGC \\
\hline & Reverse: GGCATGGACTGTGGTCATGAG \\
\hline
\end{tabular}

AC010970, AC010970.1; APBA2, amyloid $\beta$ precursor protein binding family A member 2; BRD2, bromodomain containing 2; DDX52, DExD-box helicase 52; FCAR, Fc fragment of IgA receptor; HLA-DQA1, major histocompatibility complex class II DQ $\alpha 1$; MDGA1, MAM domain containing glycosylphosphatidylinositol anchor 1; MMLT6, MLLT6 PHD finger containing; PIP4K2B, phosphatidylinositol-5-phosphate 4-kinase type $2 \beta$; WASF3, WAS protein family member 3 .

Adjusted $\mathrm{P}<0.05$ (Student's t-test with Benjamini-Hochberg false discovery rate adjustment) was used as the threshold for significant DEGs. DEGs were analyzed by enrichment analyses to detect over-represented functional terms present in the genomic background. Gene ontology (GO) analysis was performed using the GO-seq package (bioconductor.org/packages/release/bioc/html/goseq.html) in R3.2.5 software, in 
Table III. Results of alignment from RNA sequencing libraries.

\begin{tabular}{lcccc}
\hline Sample ID & Total reads & Uniquely mapped paired read $(\%)$ & Uniquely mapped unpaired read $(\%)$ & GC rate, $\%$ \\
\hline Control 1 & 45805780 & $24193674(52.8180)$ & $551484(1.2040)$ \\
Control 2 & 53239568 & $36388990(68.3495)$ & $995799(1.8704)$ & $479564(0.9630)$ \\
NRDS1 & 49799542 & $19819046(39.7977)$ & $573098(1.0812)$ & 58.8 \\
NRDS2 & 53005504 & $26210282(49.4482)$ & $514729(0.9585)$ & 56.7 \\
NRDS3 & 53700026 & $23724822(44.1803)$ & $580637(1.1446)$ & 56.3 \\
NRDS4 & 50728662 & $24525002(48.3455)$ &
\end{tabular}

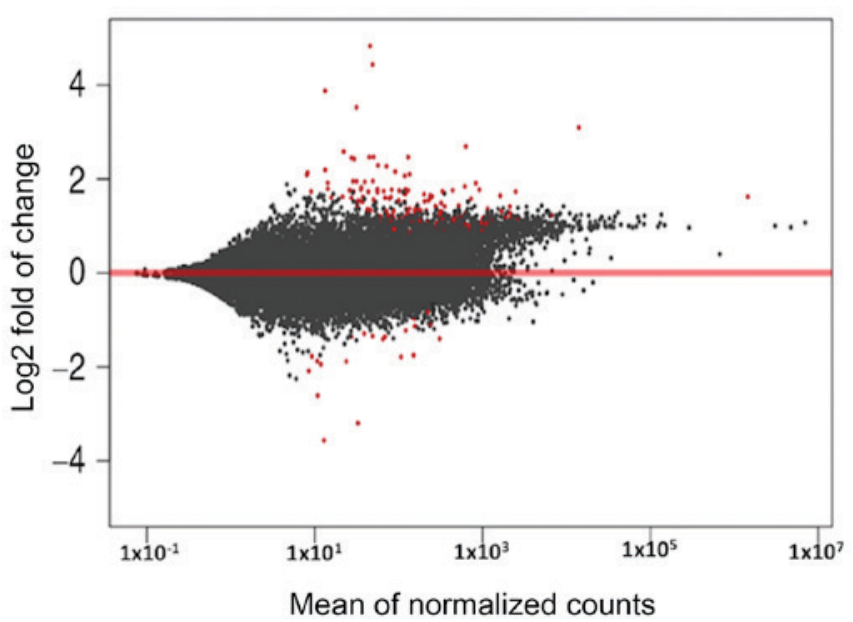

Figure 1. MA plot of all significantly differentially expressed genes between the neonatal respiratory distress syndrome and control groups, marked as red dots $(\mathrm{P}<0.05 ; \log 2 \mathrm{FC}>1)$.

which gene length bias was corrected. Kyoto Encyclopedia of Genes and Genomes (KEGG) pathway analysis was performed using KOBAS 3.0 software (kobas.cbi.pku.edu.cn).

Reverse transcription-quantitative polymerase chain reaction (RT-qPCR) analysis. Total RNAs were isolated and quality controlled using protocols from Illumina, Inc. All cDNAs were synthesized using Superscript III (Invitrogen; Thermo Fisher Scientific, Inc., Waltham, MA, USA). The top five overand underexpressed genes were selected for the RT-qPCR assay. All RT-qPCR primers are presented in Table II. All qPCR reactions were performed on a Roche Lightcycler 480 PCR system (Roche Applied Science, Penzberg, Germany) using Toyobo Thunderbird SYBR RT-qPCR Mix (Toyobo Life Science, Osaka, Japan), with three technical repeats. The amplification procedure was as follows: $95^{\circ} \mathrm{C}$ for $5 \mathrm{~min}$, followed by 40 cycles of $95^{\circ} \mathrm{C}$ for $10 \mathrm{sec}$ and $60^{\circ} \mathrm{C}$ for $20 \mathrm{sec}$. Relative quantification of target genes was performed using the $2^{-\Delta \Delta \mathrm{Cq}}$ method with GAPDH as a reference gene (13). Pearson correlation was used to calculate the association between RNA sequence and RT-qPCR (14).

\section{Results}

RNA-seq libraries. To replicate the results, control samples were obtained from two patients and NRDS samples from four patients. In total, $>300$ million reads were acquired from

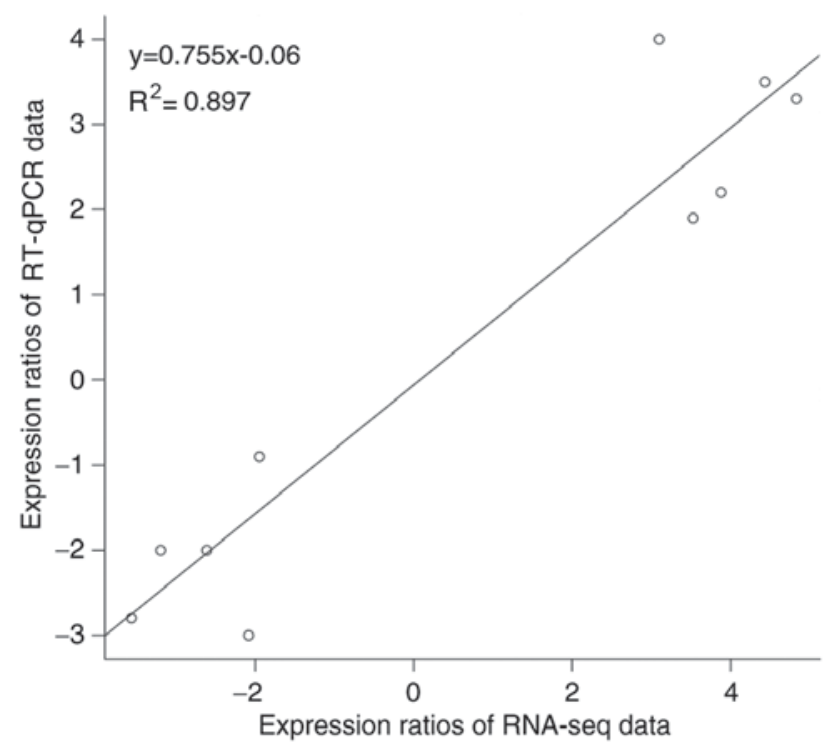

Figure 2. Correlation between RNA-seq and RT-qPCR data. RNA-seq, RNA sequencing; RT-qPCR, reverse transcription-quantitative polymerase chain reaction.

the six samples. The average percentage of uniquely mapped paired reads was $50.45 \%$ and the average GC percentage was $53.7 \%$. Detailed alignment data are presented in Table III.

Identification of DEGs. Compared with the control group, 80 genes were differentially expressed in the NRDS group. Among them, 69 genes were upregulated and 11 were downregulated. Fig. 1 illustrates all significant DEGs in an MA plot. The top 10 upregulated and downregulated genes (in terms of $\log _{2} \mathrm{FC}$ ) are presented in Table IV.

In addition, the $\log 2$ fold change of 10 genes obtained by RNA-seq and RT-qPCR analysis was compared to validate the DEGs. There was a statistically significant Pearson correlation $\left(\mathrm{R}^{2}=0.897\right)$ between the expression levels measured by RNA-seq and RT-qPCR (Figs. 2 and 3).

GO and KEGG analysis. The mRNA-seq data set comparison to KEGG (12 pathways; Fig. 4) and GO (105 gene sets; top 20 presented in Table V) pathways highlighted the up- and downregulation of three groupings of genes associated with biological function: Cellular processes; innate immune response; and pathogen recognition response. Of the 12 pathways, five of them were associated with immunological mechanisms: The tumor necrosis factor (TNF) signaling pathway (Fig. 5); influenza A 
Table IV.Top 10 differentially expressed genes in the neonatal respiratory distress syndrome group compared with the control group.

\begin{tabular}{llll}
\hline Gene & Log $_{2} \mathrm{FC}$ & P-value & \\
\hline Upregulated & & & \multicolumn{1}{c}{ Description } \\
PIP4K2B & 4.83 & $1.96 \times 10^{-24}$ & Phosphatidylinositol-5-phosphate 4-kinase type 2 $\beta$ \\
FCAR & 4.43 & $5.43 \times 10^{-19}$ & Fc fragment of IgA receptor \\
MLLT6 & 3.87 & $1.19 \times 10^{-13}$ & PHD finger domain containing \\
DDX52 & 3.52 & $2.82 \times 10^{-11}$ & DExD-box helicase 52 \\
AC010970.2 & 3.09 & $4.95 \times 10^{-10}$ & AC010970.2 \\
HBD & 2.69 & $1.49 \times 10^{-08}$ & Hemoglobin subunit $\delta$ \\
GPR84 & 2.58 & $2.65 \times 10^{-07}$ & G protein-coupled receptor 84 \\
MTRNR2L12 & 2.47 & $1.05 \times 10^{-07}$ & MT-RNR2-like 12 \\
LRRC37A2 & 2.46 & $4.07 \times 10^{-07}$ & Leucine rich repeat containing 37 member A2 \\
Downregulated & & & \\
APBA2 & -3.56 & $4.91 \times 10^{-11}$ & Amyloid $\beta$ precursor protein binding family A member 2 \\
BRD2 & -3.19 & $2.45 \times 10^{-13}$ & Bromodomain containing 2 \\
HLA-DQA1 & -2.61 & $1.04 \times 10^{-06}$ & Major histocompatibility complex, class II, DQ $\alpha$ 1 \\
MDGA1 & -2.08 & $1.10 \times 10^{-04}$ & MAM domain containing glycosylphosphatidylinositol anchor 1 \\
WASF3 & -1.94 & $1.89 \times 10^{-04}$ & WAS protein family member 3 \\
COL10A1 & -1.88 & $1.84 \times 10^{-05}$ & Collagen type X $\alpha$ 1 chain \\
GAN & -1.78 & $8.67 \times 10^{-11}$ & Gigaxonin \\
LRRN3 & -1.39 & $2.48 \times 10^{-06}$ & Leucine rich repeat neuronal 3 \\
GATA3 & -1.36 & $9.47 \times 10^{-05}$ & GATA binding protein 3 \\
CSF1R & -1.13 & $1.4 \times 10^{-04}$ & Colony stimulating factor 1 receptor \\
\hline
\end{tabular}

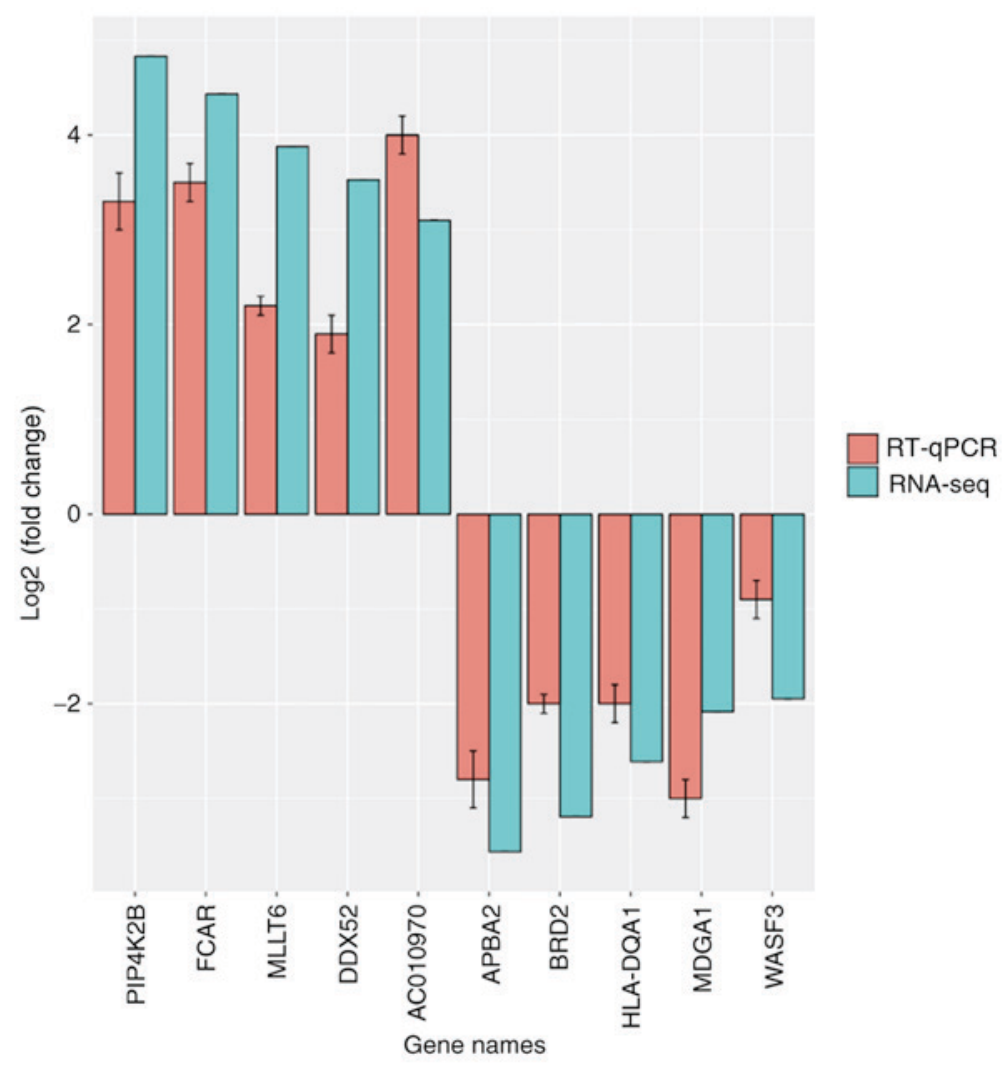

Figure 3. Expression of the top five over- and underexpressed genes, detected by RNA-seq (blue) and RT-qPCR (red). RNA-seq, RNA sequencing; RT-qPCR, reverse transcription-quantitative polymerase chain reaction; PIP4K2B, phosphatidylinositol-5-phosphate 4-kinase type $2 \beta$; FCAR, Fc fragment of IgA receptor; MLLT6, MLLT6 PHD finger containing; DDX52, DExD-box helicase 52; AC070970, AC010970.1; APBA2, amyloid $\beta$ precursor protein binding family A member 2; BRD2, bromodomain containing 2; HLA-DQA1, major histocompatibility complex class II DQ $\alpha$ 1; MDGA1, MAM domain containing glycosylphosphatidylinositol anchor 1; WASF3, WAS protein family member 3. 
Table V. Top 20 enriched GO terms among significantly differentially expressed genes.

\begin{tabular}{ll}
\hline GO ID & \multicolumn{1}{c}{ Title } \\
\hline GO:0006260 & DNA replication \\
GO:0001878 & Response to yeast \\
GO:0060045 & $\begin{array}{l}\text { Positive regulation of } \\
\text { cardiac muscle cell } \\
\text { proliferation }\end{array}$ \\
\hline
\end{tabular}

GO:0032094 Response to food

GO:0051726 Regulation of cell cycle

GO:0042246 Tissue regeneration

GO:0042742 Defense response to bacterium

GO:0060333 Interferon-gammamediated signaling pathway

GO:0032201 Telomere maintenance via semi-conservative replication

GO:0045003 Double-strand break repair via synthesisdependent strand annealing

GO:0000077 DNA damage checkpoint

GO:0006513 Protein monoubiquitination

GO:0006953 Acute-phase response

GO:0000722 Telomere maintenance via recombination

GO:0050829 Defense response to Gram-negative bacterium

GO:0006955 Immune response

GO:0097192 Extrinsic apoptotic signaling pathway in absence of ligand

GO:0009408 Response to heat
The cellular metabolic process in which a cell duplicates one or more molecules of DNA.

Any process that results in a change in state or activity of a cell or an organism as a result of a stimulus from a yeast species.

Any process that activates or increases the frequency, rate or extent of cardiac muscle cell proliferation.

Any process that results in a change in state or activity of a cell or an organism as a result of a food stimulus.

Any process that modulates the rate or extent of progression through the cell cycle.

The regrowth of lost or destroyed tissues.

Reactions triggered in response to the presence of a bacterium that act to protect the cell or organism.

A series of molecular signals initiated by the binding of interferon-gamma to a receptor on the surface of a cell, and ending with regulation of a downstream cellular process.

The process in which telomeric DNA is synthesized semi-conservatively by the conventional replication machinery and telomeric accessory factors as part of cell cycle DNA replication.

Synthesis-dependent stand annealing is a major mechanism of double-strand break repair in mitosis that allows for the error-free repair of a double-strand break without the exchange of adjacent sequences.

A cell cycle checkpoint that regulates progression through the cell cycle in response to DNA damage. Addition of a single ubiquitin group to a protein.

An acute inflammatory response that involves non-antibody proteins whose concentrations in the plasma increase in response to infection or injury of homeothermic animals.

Any recombinational process that contributes to the maintenance of proper telomeric length.

Reactions triggered in response to the presence of a Gram-negative bacterium that act to protect the cell or organism.

Any immune process that functions in the calibrated response of an organism to a potential internal or invasive threat.

A series of molecular signals in which a signal is conveyed from the cell surface to trigger the apoptotic death of a cell.

Any process that results in a change in state or activity of a cell or an organism (in terms of movement, secretion, enzyme production, gene expression, etc.) as a result of a heat stimulus, a temperature stimulus above the optimal temperature for that organism.
No. of genes P-value

5

0.000278

2

0.000873

2

0.001500

2

0.001750

4

0.001760

2

0.002900

3

0.003520

3

0.004290

2

0.005140

2

0.006020 
Table V. Continued.

\begin{tabular}{|c|c|c|c|c|}
\hline GO ID & Title & Description & No. of genes & P-value \\
\hline GO:0032508 & DNA duplex unwinding & $\begin{array}{l}\text { The process in which interchain hydrogen bonds } \\
\text { between two strands of DNA are broken or } \\
\text { 'melted', generating a region of unpaired single strands. }\end{array}$ & 2 & 0.018100 \\
\hline GO:0001504 & Neurotransmitter uptake & $\begin{array}{l}\text { The directed movement of neurotransmitters into } \\
\text { neurons or glial cells. }\end{array}$ & 1 & 0.022300 \\
\hline
\end{tabular}

GO, gene ontology.

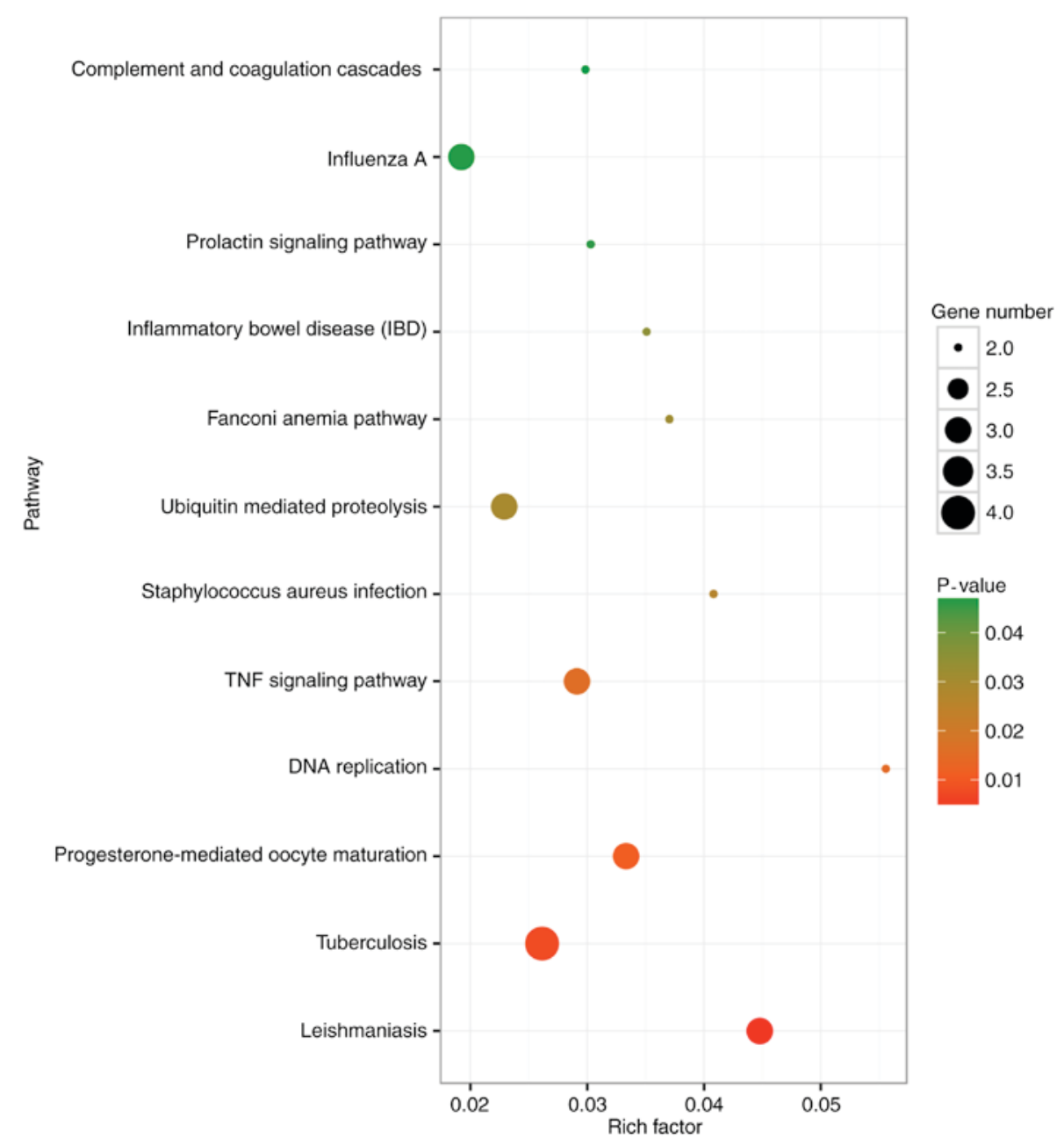

Figure 4. Kyoto Encyclopedia of Genes and Genomes enrichment analysis of differentially expressed genes $(\mathrm{P}<0.05)$.

(Fig. 6); leishmaniasis (Fig. 7); Staphylococcus aureus infection (Fig. 8); and tuberculosis (Fig. 9). Suppressor of cytokine signaling 3, mitogen-activated protein kinase 14 and major histocompatibility complex II are overexpressed in these pathways; all three of these genes are involved in immune processes.

Within the groupings of cellular processes, the DNA replication and Fanconi anemia pathways were downregulated by 93 and 318 nuclear receptor (NR) genes, respectively, in response to NRDS pathogenesis compared with the control group. By contrast, the cellular proteolysis by ubiquitination and hormone signaling pathways were upregulated by 104 and 82 NR genes, respectively.

\section{Discussion}

The phenotype of NRDS is not concluded from a single symptom; rather it is derived from numerous cumulative factors which precipitate NRDS. RNA-seq is an useful tool to illustrate the physiological responses to multiple factors which contribute to the manifestation of NRDS. The following identifies a number of mechanisms observed in the RNA-seq results, which are consistent with those reported in the process of ARDS.

In a case-control study reported by Molnar et al (15), increased levels of ubiquitin specific peptidase 10 (USP10) were observed in the ARDS group. In the present study, USP1 was observed 

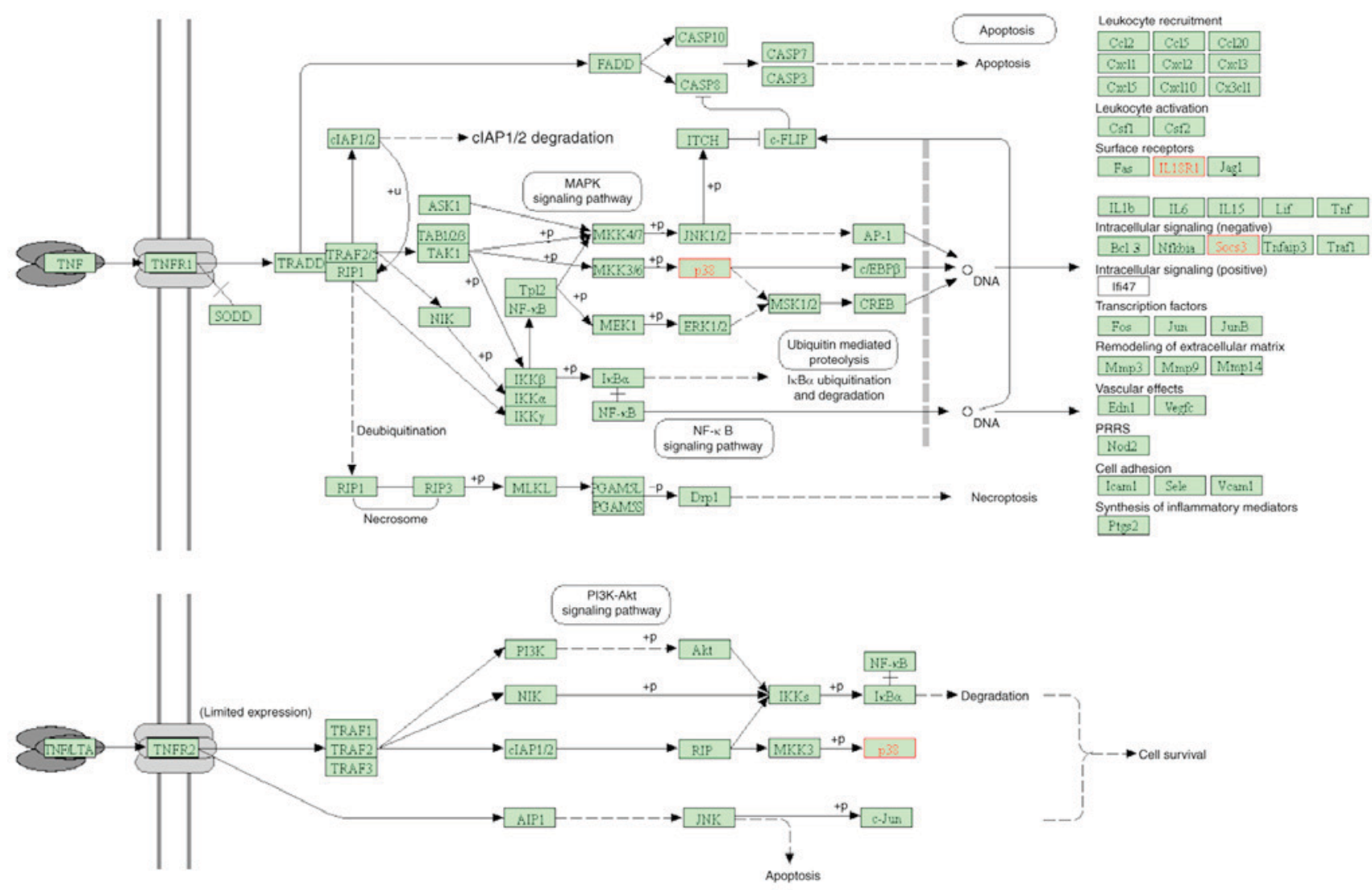

Figure 5. Differentially expressed genes (red) in the neonatal respiratory distress syndrome group compared with the control group in the TNF signaling pathway. TNF, tumor necrosis factor; SOCS3, suppressor of cytokine signaling 3; IL18R1, interleukin 18 receptor 1; p38, mitogen-activated protein kinase 14.

to be increased in the NRDS group (data not shown). As USP proteins are involved in autophagy, it may be hypothesized that ARDS and NRDS exhibit similar processes of autophagy.

A recent study by Qiao et al (16) illustrated that the ubiquitin-proteasome pathway (UPP) targeted signal transducer and activator of transcription 6 and further regulated Thelper cells 2 and 9; this regulation may serve as an ideal target for mitigating the underlying pathology of allergic diseases, particularly asthma. Dysregulation and chronic overexpression of the UPP ligase systems and other ubiquitinated kinase protein components may cause inflammation of the bronchi and promote the pathophysiology of NRDS. Majetschak et al (17) demonstrated that ubiquitin-activated 20S core proteasome particles in bronchoalveolar lavage fluid were markedly increased following lung contusions. The proteolytic cleavage of functional proteasome complexes and additional post-protein processing observed within the alveolar space following lung injury may be associated with the pathogenesis of NRDS. It is unknown how cleavage and post-regulation of proteins involved in lung edema clearance interact with essential surfactant proteins, which may further enhance the NRDS pathological cascade.

Inhibition of the ubiquinated protein kinase myristoylated alanine rich protein kinase $\mathrm{C}$ substrate (MARCKS) serves a role in mitigating excess pro-inflammatory cytokines release from neutrophils following lipopolysaccharide (LPS) from Escherichia coli and the activation of Toll-like receptor (TLR)2, TLR4 and TLR9 (18). The results of this previous study illustrated correct and incorrect post-transcriptional regulation. When organisms were treated with the protein myristoylated N-terminal sequence, an inhibitor of MARCKS, neutrophil migration was mediated; marked alterations were observed in the reduction of mRNA expression associated with LPS-challenged interleukin- 8 and TNF- $\alpha$.

The assessment of various lung cellular responses to numerous environmental factors, by assessing mRNA-seq associated with NRDS, is a viable and sophisticated way to characterize NRDS and ARDS. Assessing the progression of various symptoms towards the onset of diseases may help to further characterize cellular responses associated with disease and to further elucidate the underlying pathogenesis of diseases. A previous study assessed how alterations at the mRNA level affect how transcriptional gene regulation is moderated prior to the production and translation of proteins (15). Gao and Barnes (19) performed a meta-analysis using RNA-seq data of 83 lung-specific genes; only 62 of the genes were required to code for proteins necessary for lung development and pathogenesis. Specifically, in ARDS there are 21 principal genes of interest to target that are associated with the physiological onset of ARDS and similar acute lung injuries.

Due to the hydrophobic nature of surfactants, they are able to keep the alveoli dry and allow for optimal oxygen-blood exchange. Dysregulation of surfactant phospholipids has been previously demonstrated to be associated with alveolar instability and an increased patient risk of developing NRDS (20). 


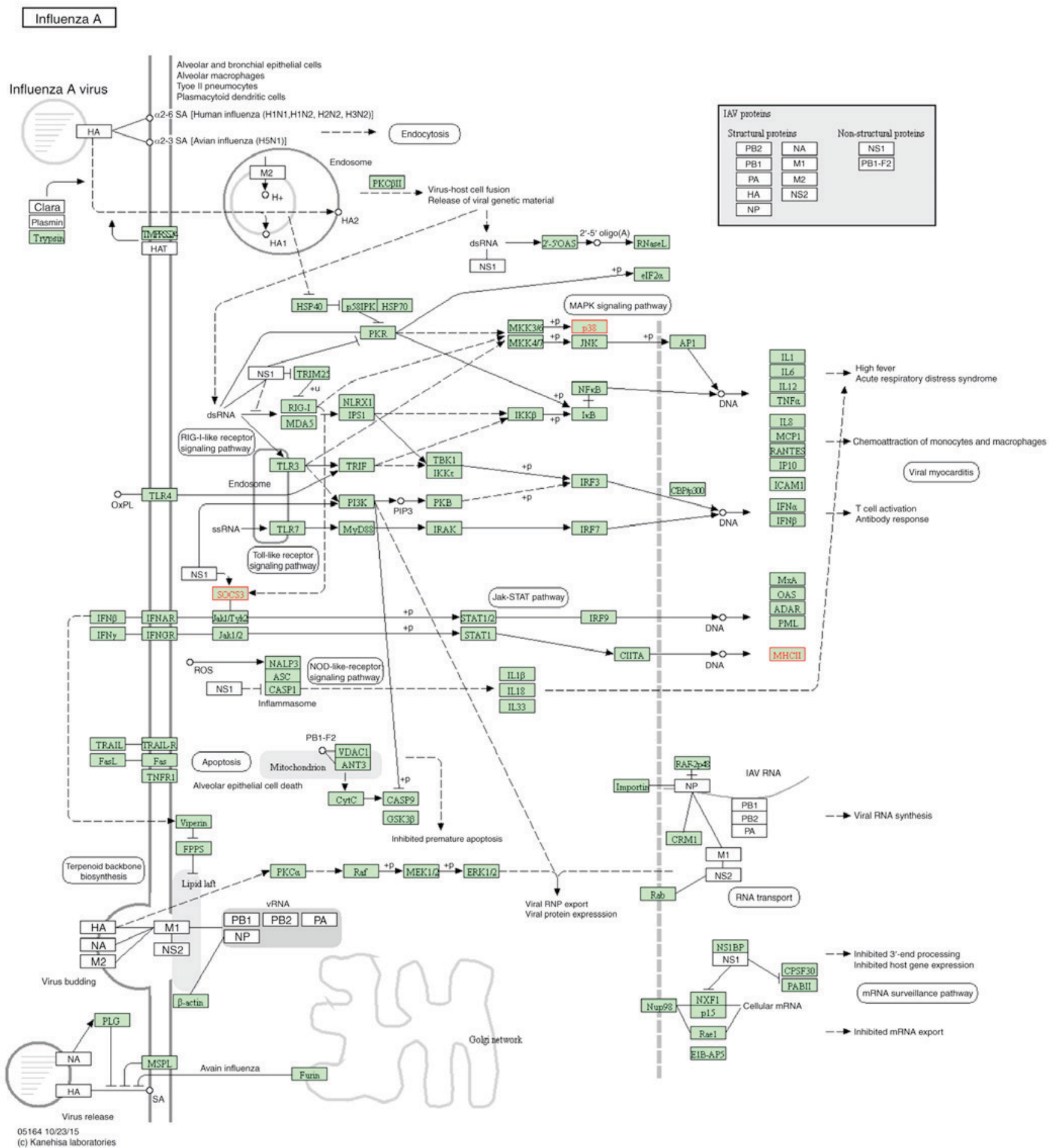

Figure 6. Differentially expressed genes (red) in the neonatal respiratory distress syndrome group compared with the control group in the influenza A pathway. SOCS3, suppressor of cytokine signaling 3; MHCII, major histocompatibility complex II; p38, mitogen-activated protein kinase 14.

Translational regulation of gene families has essential roles in maintaining lung homeostasis, including surfactant-associated proteins (SFTPs; SFTPA1, SFTPA2, SFTPC and SFTPD) which serve a role in understanding the pathology of NRDS $(21,22)$.

In a study by D'Ovidio et al (23), clinicians assessed the mortality associated with lung transplants and various mRNA levels among different experimental groups and their surfactant protein polymorphism (SP-A, SP-B, SP-C and SP-D) expression levels. The pilot study demonstrated notable mortality rates in patients; however, those with increased mRNA expression of SP generally exhibited a decreased incidence of allograft rejection by the innate immune system, thus illustrating a marked difference in lung transplant mortality associated with surfactants. Additionally, Silva et al (24) observed that the application of the anti-inflammatory agent LASSBio596 may be applied to ARDS mouse models to increase survival rates by mitigating macrophages, neutrophils and transforming growth factor- $\beta$ by increasing surfactant yield, which in turn reduced surface tension. By maintaining 


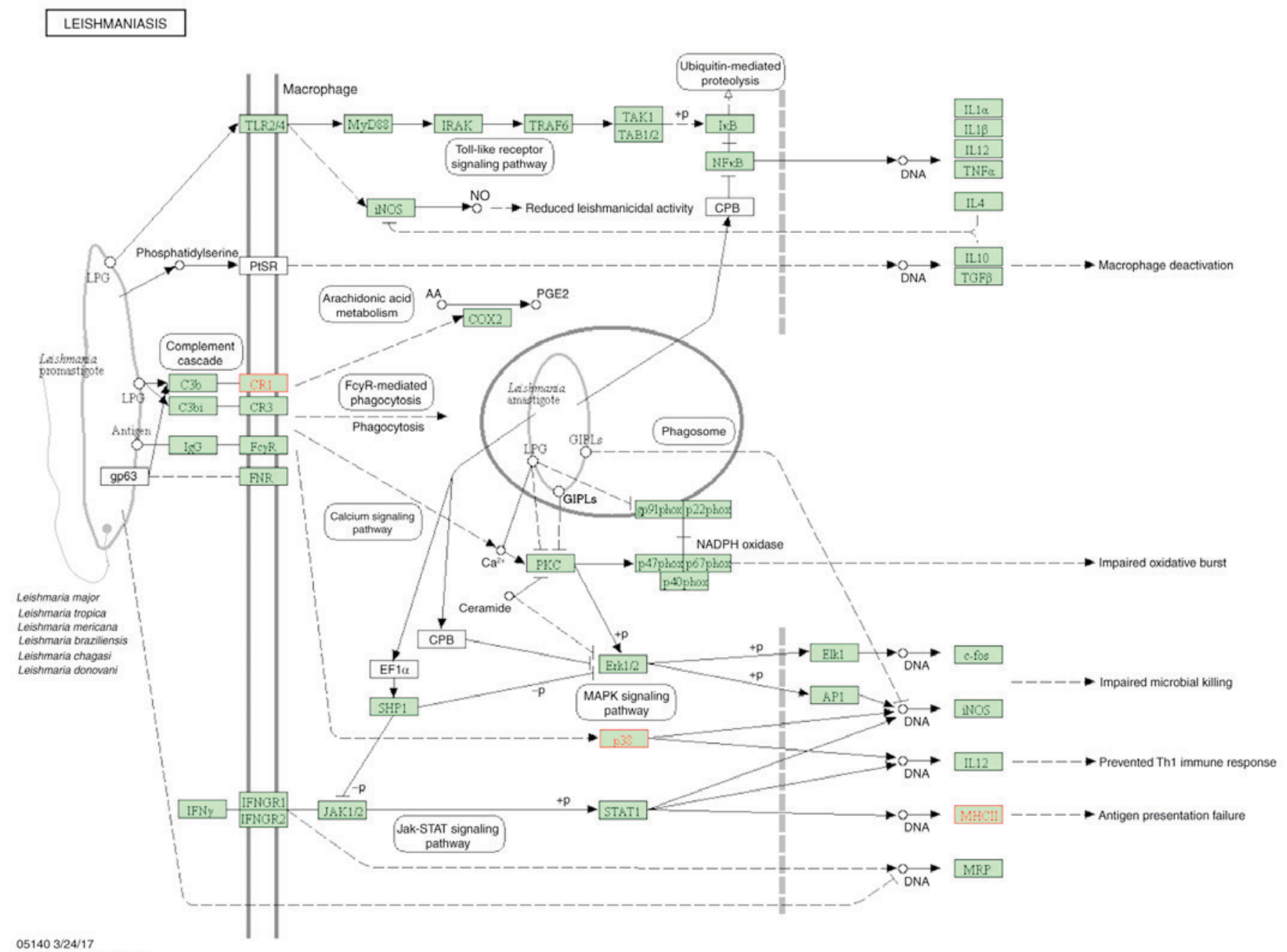

$051403 / 24 / 17$

Figure 7. Differentially expressed genes (red) in the neonatal respiratory distress syndrome group compared with the control group in the Leishmaniasis pathway. MHCII, major histocompatibility complex II; CR1, complement C3b/C4b receptor 1; p38, mitogen-activated protein kinase 14.

elasticity and surfactant alterations in lung mechanics, the authors were able to counteract degradation immediately following lung injury leading to ARDS.

The removal of surfactant proteins leads to substantial alterations in the alveoli-capillary barrier, leading to the serious phenotype of sepsis. Pathogenesis-induced sepsis of the peripheral lung is denoted by inflammation associated with the presence of the microbial metabolite lactate, which is a standard lung biomarker of sepsis (25). In response to a microbial presence in the form of adhesion or byproducts, the innate immune system is the first to mount a response. In order to further assess the biomarkers of NRDS, Ware et al (26) demonstrated that SP-D overexpression is an effective marker of sepsis prognosis; increased expression of this protein from alveolar epithelial type II cells is associated with increased mortality.

In the grouping of gene families associated with the innate immune response, it has been reported that all four pathways, complement and coagulation cascades, tumor TNF signaling, inflammatory bowel disease and prolactin signaling, were upregulated by 50-67 genes in response to NRDS. In the case of pneumonia, pro-inflammatory cytokines and systemic inflammation, the host-associated response will lead to increased TNF expression followed by dysregulation of lung-induced apoptosis by neutrophils (27). Proteinase-activated cascades following lung injury promote the activation of the coagulation response that begins the innate inflammatory response and the additional pro-inflammatory factors, including TNF (28). Macrophage-derived TNF has been demonstrated to further provoke lung injury by stimulating p55 receptor-mediated death signaling; this further exacerbates injury by increasing lung neutrophils and leads to the deleterious phenotype of ARDS (29).

Upstream activation of TNF- $\alpha$ within the lung periphery is an essential mediator of neutrophils, and the pathology of NRDS and ARDS. Plasma TNF- $\alpha$ levels have been observed to be increased in patients with NRDS compared with controls (30). A study performed in mice by Lomas-Neira et al (31) demonstrated that the transfer of TNF- $\alpha$ to mice lacking neutrophils did not stimulate the progression of lung injury. By comparison, mice lacking neutrophils expressing TNF- $\alpha$ displayed reduced acute lung injury. These previous results suggested that TNF- $\alpha$ expression in neutrophils is an underlying factor the promotes the progression of the pathogenesis from the initial lung-injury into a shock-primed innate immune response.

In response to colonization by pathogenic organisms, including Streptococcus pneumoniae, proteinase-activated receptor 1 serves a role in splicing for the activation of 
STAPHYLOCOCCUS AUREUS INFECTION

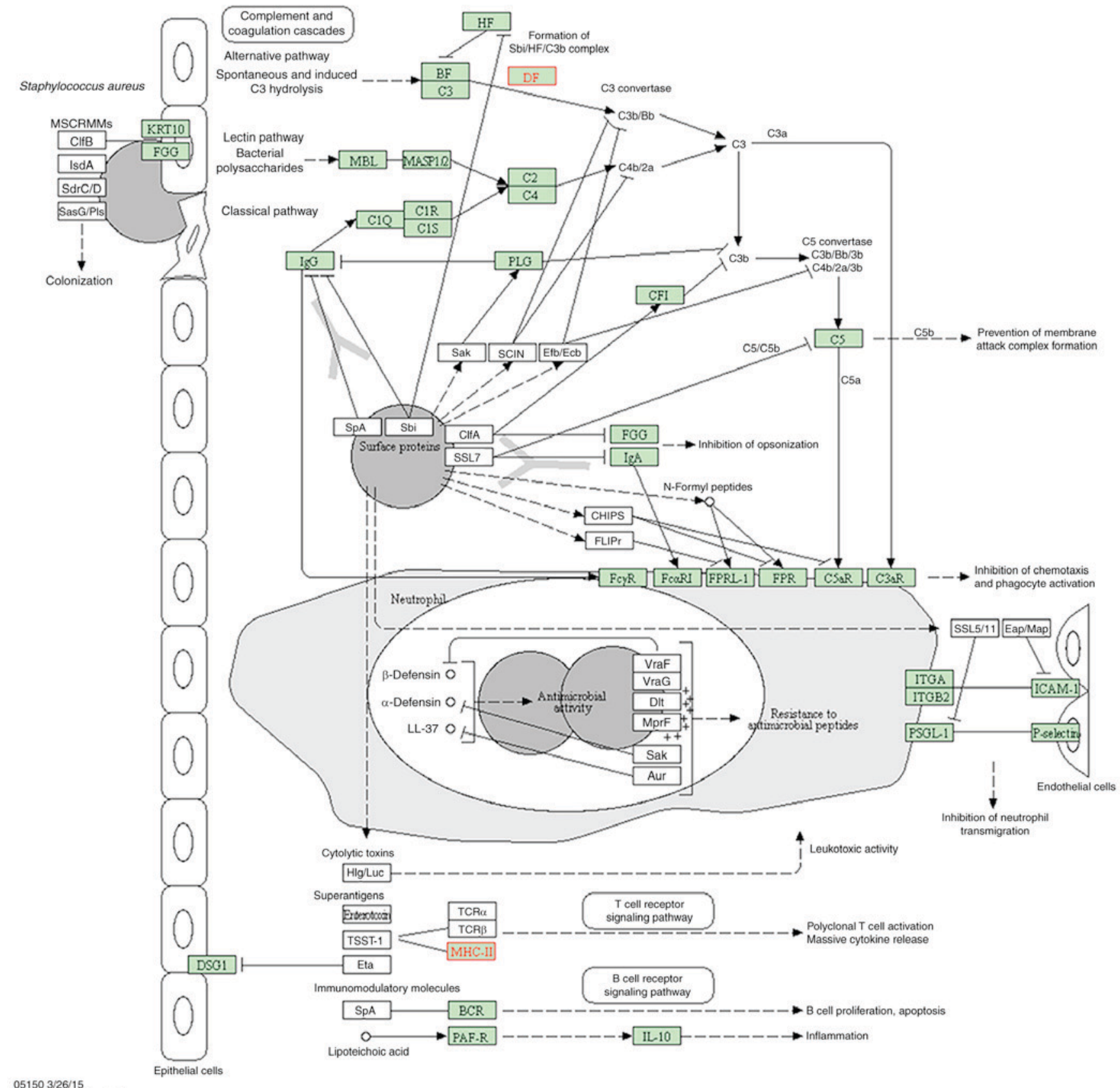

Figure 8. Differentially expressed genes (red) in the neonatal respiratory distress syndrome group compared with the control group in the Staphylococcus aureus infection pathway. DF, complement factor D; MHCII, major histocompatibility complex II.

coagulation pathways leading to inflammation; thus, beginning the cascade of neutrophilic pro-inflammatory responses to invasive pathogens, excess neutrophil recruitment contributes to lung injury through alveolar surfactant barrier disruption and alveolar leakage (18).

The pathogen recognition response for NRDS KEGG pathways all had upregulated gene families in the present study; this was the case for all except Staphylococcus aureus infection, which was downregulated by 10 NR genes. Upregulated gene families included leishmaniasis, tuberculosis, and influenza A, and the number of genes involved in these three groups were 3, 4 and 3, respectively. Microbial proliferation and sepsis following acute lung injury is a primary contributor to the cascade and severity of NRDS. Lv et al (32) demonstrated through transcriptomic analysis that genes associated with foreign pathogens (NLR family pyrin domain containing 3 and S100 calcium binding protein A8/A9) were markedly upregulated in response to microbial LPS.

In addition to sepsis, other factors not involved with the innate immune response or surfactants may contribute to NRDS and ARDS. For example, Lv et al (32) demonstrated, through systematic mRNA-seq of ARDS, a significant upregulation of 122 genes and a downregulation of 91 genes associated with essential protein functions, including the mitotic cell cycle. 


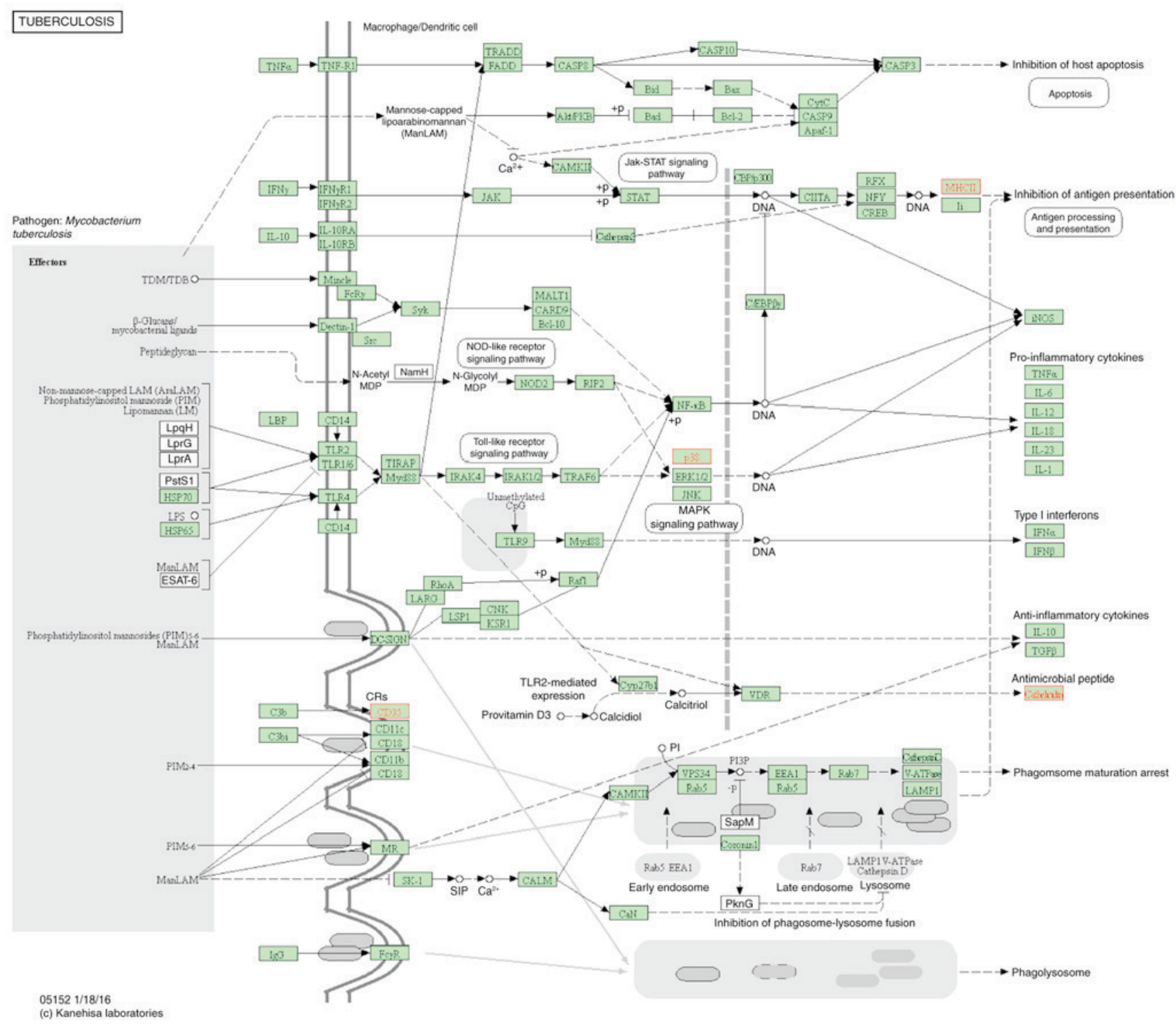

Figure 9. Differentially expressed genes (red) in the neonatal respiratory distress syndrome group compared with the control group in the tubercuosis pathway. p38, mitogen-activated protein kinase 14; MHCII, major histocompatibility complex II; CD35, complement C3b/C4b receptor 1.

Specifically, the investigators noted that cyclin (CCNB)1 and CCNB2, which serve a role in cell cycle regulation, may be associated with the onset of ARDS.

A previous study of the human microbiome indicated dysbiosis within the human system, typically leading to a dysregulation of host responses to various systemic challenges; in particular, these challenges threaten lung homeostasis and provoke the perpetual inflammation associated with invasive pathogenic microbiota (33). Invasion, persistence and colonization of foreign microbiota challenge the host immune system to further provoke ARDS; the proliferation of microbiota and heightened responses to challenge further provoke the accumulated lung injury to develop into sepsis (34). Infections with and the persistence of numerous viral strains and microbiota, including influenza A (35), Mycobacterium tuberculosis (36), Staphylococcus aureus/H1N1 strain of the influenza virus (37) and multi-drug resistant Acinetobacter baumannii (38).

Dysregulation of TNF and the coagulation cascade pathways may be associated with ubiquitin-mediated kinase activation in the case of proteasome activated lysis, which may activate cascades leading to neutrophil over-stimulation and eventual dysregulated lung damage. In conclusion, the mechanisms leading to a specific NRDS phenotype are complex, although mRNA-seq as a tool may be used to elucidate genomic response to lung-induced stress. Consequently, cascading immune mechanisms associated with the dysregulation of one aspect of the immune response may accumulate progressively towards NRDS.

\section{Acknowledgements}

The present study was supported by the National Natural Science Foundation of China (grant no. gjzr81260107) and the Inner Mongolia Natural Science Foundation (grant no. 2011MS1111).

\section{References}

1. Angus DC, Linde-Zwirble WT, Clermont G, Griffin MF and Clark RH: Epidemiology of neonatal respiratory failure in the United States: Projections from California and New York. Am J Respir Crit Care Med 164: 1154-1160, 2001. 
2. Santos C, Ferrer M, Roca J, Torres A, Hernández C and Rodriguez-Roisin R: Pulmonary gas exchange response to oxygen breathing in acute lung injury. Am J Respir Crit Care Med 161: 26-31, 2000.

3. Doyle IR, Bersten AD and Nicholas TE: Surfactant proteins-A and $-\mathrm{B}$ are elevated in plasma of patients with acute respiratory failure. Am J Respir Crit Care Med 156: 1217-1229, 1997.

4. Dolinay T, Kim YS, Howrylak J, Hunninghake GM, An CH, Fredenburgh L, Massaro AF, Rogers A, Gazourian L, Nakahira K, et al: Inflammasome-regulated cytokines are critical mediators of acute lung injury. Am J Respir Crit Care Med 185: 1225-1234, 2012.

5. Speer CP, Robertson B, Curstedt T, Halliday HL, Compagnone D, Gefeller O, Harms K, Herting E, McClure G, Reid M, et al: Randomized European multicenter trial of surfactant replacement therapy for severe neonatal respiratory distress syndrome: Single versus multiple doses of Curosurf. Pediatrics 89: 13-20, 1992.

6. Enhorning G, Shennan A, Possmayer F, Dunn M, Chen CP and Milligan J: Prevention of neonatal respiratory distress syndrome by tracheal instillation of surfactant: A randomized clinical trial. Pediatrics 76: 145-153, 1985.

7. Thawer S, Auret J, Schnoeller C, Chetty A, Smith K, Darby M, Roberts L, Mackay RM, Whitwell HJ, Timms JF, et al: Surfactant protein-D is essential for immunity to helminth infection. PLoS Pathog 12: e1005461, 2016.

8. de Guevara YL, Hidalgo OB, Santos SS, Brunialti MK, Faure R and Salomao R: Effect of natural porcine surfactant in Staphylococcus aureus induced pro-inflammatory cytokines and reactive oxygen species generation in monocytes and neutrophils from human blood. Int Immunopharmacol 21: 369-374, 2014.

9. Bernard GR, Artigas A, Brigham KL, Carlet J, Falke K, Hudson L, Lamy M, Legall JR, Morris A and Spragg R: The American-European Consensus Conference on ARDS Definitions, mechanisms, relevant outcomes and clinical trial coordination. Am J Respir Crit Care Med 149: 818-824, 1994.

10. Khadaroo RG and Marshall JC: ARDS and the multiple organ dysfunction syndrome. Common mechanisms of a common systemic process. Crit Care Clin 18: 127-141, 2002.

11. Sweet DG, Carnielli V, Greisen G, Hallman M, Ozek E, Plavka R, Saugstad OD, Simeoni U, Speer CP, Halliday HL, et al: European consensus guidelines on the management of neonata respiratory distress syndrome in preterm infants-2010 update. Neonatology 97: 402-417, 2010.

12. Trapnell C, Williams BA, Pertea G, Mortazavi A, Kwan G, van Baren MJ, Salzberg SL, Wold BJ and Pachter L: Transcript assembly and quantification by RNA-Seq reveals unannotated transcripts and isoform switching during cell differentiation. Nat Biotechnol 28: 511-515, 2010

13. Livak KJ and Schmittgen TD: Analysis of relative gene expression data using real-time quantitative PCR and the 2(-Delta Delta $\mathrm{C}(\mathrm{T})$ ) method. Methods 25: 402-408, 2001

14. Häne BG, Jäger K and Drexler HG: The Pearson product-moment correlation coefficient is better suited for identification of DNA fingerprint profiles than band matching algorithms. Electrophoresis 14: 967-972, 1993.

15. Molnar CS, Sárvári M, Vastagh C, Maurnyi C, Fekete C, Liposits Z and Hrabovszky E: Altered gene expression profiles of the hypothalamic arcuate nucleus of male mice suggest profound developmental changes in peptidergic signaling. Neuroendocrinology 103: 369-382, 2016.

16. Qiao G, Ying H, Zhao Y, Liang Y, Guo H, Shen H, Li Z, Solway J, Tao E, Chiang YJ, et al: E3 ubiquitin ligase Cbl-b suppresses proallergic $\mathrm{T}$ cell development and allergic airway inflammation. Cell Rep 6: 709-723, 2014.

17. Majetschak M, Sorell LT, Patricelli T, Seitz DH and Knoferl MW: Detection and possible role of proteasomes in the bronchoalveolar space of the injured lung. Physiol Res 58: 363-372, 2009.

18. José RJ, Williams AE, Mercer PF, Sulikowski MG, Brown JS and Chambers RC: Regulation of neutrophilic inflammation by proteinase-activated receptor 1 during bacterial pulmonary infection. J Immunol 194: 6024-6034, 2015.

19. Gao L and Barnes KC: Recent advances in genetic predisposition to clinical acute lung injury. Am J Physiol Lung Cell Mol Physiol 296: L713-L725, 2009.

20. Gregory TJ, Longmore WJ, Moxley MA, Whitsett JA, Reed CR, Fowler AA III, Hudson LD, Maunder RJ, Crim C and Hyers TM: Surfactant chemical composition and biophysical activity in acute respiratory distress syndrome. J Clin Invest 88: 1976-1981, 1991.
21. Melton KR, Nesslein LL, Ikegami M, Tichelaar JW, Clark JC, Whitsett JA and Weaver TE: SP-B deficiency causes respiratory failure in adult mice. Am J Physiol Lung Cell Mol Physiol 285: L543-L549, 2003.

22. Bridges JP, Wert SE, Nogee LM and Weaver TE: Expression of a human surfactant protein $\mathrm{C}$ mutation associated with interstitial lung disease disrupts lung development in transgenic mice. J Biol Chem 278: 52739-52746, 2003.

23. D'Ovidio F, Kaneda H, Chaparro C, Mura M, Lederer D, Di Angelo S, Takahashi H, Gutierrez C, Hutcheon M, Singer LG, et al: Pilot study exploring lung allograft surfactant protein $\mathrm{A}$ (SP-A) expression in association with lung transplant outcome. Am J Transplant 13: 2722-2729, 2013.

24. Silva JD, de Oliveira GP, Samary Cdos S, Araujo CC, Padilha Gde A, Costa e Silva Filho F, da Silva RT, EinickerLamas M, Morales MM, Capelozzi VL, et al: Respiratory and systemic effects of LASSBio596 plus surfactant in experimental acute respiratory distress syndrome. Cell Physiol Biochem 38 821-835, 2016.

25. Li J, D'Annibale-Tolhurst MA, Adler KB, Fang S, Yin Q, Birkenheuer AJ, Levy MG, Jones SL, Sung EJ, Hawkins EC, et al: A myristoylated alanine-rich C kinase substrate-related peptide suppresses cytokine mRNA and protein expression in LPS-activated canine neutrophils. Am J Respir Cell Mol Biol 48: 314-321, 2013.

26. Ware LB, Koyama T, Zhao Z, Janz DR, Wickersham N, Bernard GR, May AK, Calfee CS and Matthay MA: Biomarkers of lung epithelial injury and inflammation distinguish severe sepsis patients with acute respiratory distress syndrome. Crit Care 17: R253, 2013.

27. Bordon J, Aliberti S, Fernandez-Botran R, Uriarte SM, Rane MJ, Duvvuri P, Peyrani P, Morlacchi LC, Blasi F and Ramirez JA: Understanding the roles of cytokines and neutrophil activity and neutrophil apoptosis in the protective versus deleterious inflammatory response in pneumonia. Int J Infect Dis 17: e76-e83, 2013.

28. Chambers RC and Scotton CJ: Coagulation cascade proteinases in lung injury and fibrosis. Proc Am Thorac Soc 9: 96-101, 2012

29. Patel BV, Wilson MR, O'Dea KP and Takata M: TNF-induced death signaling triggers alveolar epithelial dysfunction in acute lung injury. J Immunol 190: 4274-4282, 2013

30. Meng Q, Xiao DX, Zhang Z and Fu WH: Changes and clinical significance of the peripheral blood mononuclear cell NF- $\kappa$ B activity and TNF- $\alpha$ in neonatal respiratory distress syndrome. Chin J Child Health Care 4: 411-412, 2009 (In Chinese).

31. Lomas-Neira J, Perl M, Venet F, Chung CS and Ayala A: The role and source of tumor necrosis factor-alpha in hemorrhage-induced priming for septic lung injury. Shock 37: 611-620, 2012.

32. Lv X, Zhang Y, Lu W, Wang Q, Li Sy, Guo L, Qian Gs, Zhou Sw and Li Yy: Digital gene expression analysis of transcriptomes in lipopolysaccharide-induced acute respiratory distress syndrome. Clin Chim Acta 453: 182-189, 2016.

33. Dickson RP, Martinez FJ and Huffnagle GB: The role of the microbiome in exacerbations of chronic lung diseases. Lancet 384: 691-702, 2014.

34. Kitsios GD, Morowitz MJ, Dickson RP, Huffnagle GB, McVerry BJ and Morris A: Dysbiosis in the intensive care unit: Microbiome science coming to the bedside. J Crit Care 38: 84-91, 2017.

35. Short KR, Kroeze EJBV, Fouchier RAM and Kuiken T: Pathogenesis of influenza-induced acute respiratory distress syndrome. Lancet Infect Dis 14: 57-69, 2014

36. Arevalo-Lorido JC, Bureo-Dacal JC, Romero-Requena J, RamosSalado JL and Bureo-Dacal P: Tuberculosis complicated by ARDS. Ir J Med Sci 170: 209, 2001.

37. Lees NJ, Rosenberg A, Hurtado-Doce AI, Jones J, Marczin N, Zeriouh M, Weymann A, Sabashnikov A, Simon AR and Popov AF: Combination of ECMO and cytokine adsorption therapy for severe sepsis with cardiogenic shock and ARDS due to Panton-Valentine leukocidin-positive Staphylococcus aureus pneumonia and H1N1. J Artif Organs 19: 399-402, 2016

38. LinCY,Chen YM,Lin MC, Chang YP, Chao TY,Wang CC, Tsai YC, Shen LS, Li CL and Lin AS: Risk factors of multidrug-resistant Acinetobacter baumannii recurrence after successful eradication in ventilated patients. Biomed J 39: 130-138, 2016.

This work is licensed under a Creative Commons Attribution-NonCommercial-NoDerivatives 4.0 International (CC BY-NC-ND 4.0) License. 